\title{
Combining Orthology and Xenology Data in a Common Phylogenetic Tree
}

\author{
Marc Hellmuth ${ }^{1}$, Mira Michel ${ }^{2}$, Nikolai N. Nøjgaard ${ }^{3}$, David Schaller ${ }^{4,5}$, and Peter F. \\ Stadler ${ }^{4-8}$

\footnotetext{
${ }^{1}$ Department of Mathematics, Faculty of Science, Stockholm University, SE-10691 Stockholm, Sweden marc.hellmuth@math.su.se

${ }^{2}$ Faculty of Mathematics and Computer Science, Fernuniversität Hagen, Universitätsstrasse 47, D-58097 Hagen, Germany mira.michel@studium.fernuni-hagen.de

${ }^{3}$ Department of Mathematics and Computer Science, University of Southern Denmark, Odense M, Denmark nnoej10@gmail.com

${ }^{4}$ Max Planck Institute for Mathematics in the Sciences, Leipzig, Germany sdavid@bioinf.uni-leipzig.de

${ }^{5}$ Bioinformatics Group, Department of Computer Science, and Interdisciplinary Center for Bioinformatics, Universität Leipzig, Härtelstrasse 16-18, D-04107 Leipzig, Germany studla@bioinf .uni-leipzig.de

${ }^{6}$ Institute for Theoretical Chemistry, University of Vienna, Vienna, Austria

${ }^{7}$ Facultad de Ciencias, Universidad Nacional de Colombia, Bogotá, Colombia

${ }^{8}$ Santa Fe Institute, Santa Fe, New Mexico, USA
}

\begin{abstract}
A rooted tree $T$ with vertex labels $t(v)$ and set-valued edge labels $\lambda(e)$ defines maps $\delta$ and $\varepsilon$ on the pairs of leaves of $T$ by setting $\delta(x, y)=q$ if the last common ancestor lca $(x, y)$ of $x$ and $y$ is labeled $q$, and $m \in \varepsilon(x, y)$ if $m \in \lambda(e)$ for at least one edge $e$ along the path from lca $(x, y)$ to $y$. We show that a pair of maps $(\delta, \varepsilon)$ derives from a tree $(T, t, \lambda)$ if and only if there exists a common refinement of the (unique) least-resolved vertex labeled tree $\left(T_{\delta}, t_{\delta}\right)$ that explains $\delta$ and the (unique) least resolved edge labeled tree $\left(T_{\varepsilon}, \lambda_{\varepsilon}\right)$ that explains $\varepsilon$ (provided both trees exist). This result remains true if certain combinations of labels at incident vertices and edges are forbidden.
\end{abstract}

Keywords: Mathematical phylogenetics, rooted trees, binary relations, symbolic ultrametric, Fitch map, consistency

\section{Introduction}

An important task in evolutionary biology and genome research is to disentangle the mutual relationships of related genes. The evolution of a gene family can be understood as a tree $T$ whose leaves are genes and whose inner vertices correspond to evolutionary events, in particular speciations (where genomes are propagated into different lineages that henceforth evolve independently), duplications (of genes within the same genome) and horizontal gene transfer (where copies of an individual genes are transferred into an unrelated species) [4]. Mathematically, these concepts are described in terms of rooted trees $T$ with vertex labels $t$ representing event types and edge labels $\lambda$ distinguishing vertical and horizontal inheritance. On the other hand, orthology (descent from a speciation) or xenology (if the common history involves horizontal transfer events) can be regarded as binary relation on the set $L$ of genes. Given the orthology or xenology relationships, one then asks whether there exists a vertex or edge labeled tree $T$ with leaf set $L$ that "explains" the relations $[9,5]$. Here, we ask when such relational orthology and xenology data are consistent. A conceptually similar question is addressed in a very different formal setting in [15].

Instead of considering a single binary orthology or xenology relation, we consider here multiple relations of each type. This is more conveniently formalized in terms of maps that assign finite sets of labels. Two types of maps are of interest: Symbolic ultrametrics, i.e., symmetric maps determined by a label at the last common ancestor of two genes [2] generalize orthology. Fitch maps, i.e., non-symmetric maps determined by the union of labels along the path connecting two genes [12], form a generalization of xenology. For both types of maps unique least-resolved trees (minimal under edge-contraction) exist and can be constructed by polynomial time algorithms 
$[2,12]$. Here we consider the problem of finding trees that are simultaneously edge- and vertexlabeled and simultaneously explain both types of maps. We derive a simple condition for the existence of explaining trees and show that there is again a unique least-resolved tree among them. We then consider a restricted version of problem motivated by concepts of observability introduced in [17].

\section{Preliminaries}

\section{$2.1 \quad$ Trees and Hierarchies}

Let $T$ be a rooted tree with vertex set $V(T)$, leaf set $L(T) \subseteq V(T)$, set of inner vertices $V^{0}(T):=$ $V(T) \backslash L(T)$, root $\rho \in V^{0}(T)$, and edge set $E(T)$. An edge $e=\{u, v\} \in E(T)$ is an inner edge if $u, v \in V^{0}(T)$. The ancestor partial order on $V(T)$ is defined by $x \preceq_{T} y$ whenever $y$ lies along the unique path connecting $x$ and the root. We write $x \prec_{T} y$ if $x \preceq_{T} y$ and $x \neq y$. For $v \in V(T)$, we set $\operatorname{child}(v):=\left\{u \mid\{v, u\} \in E(T), u \prec_{T} v\right\}$. All trees $T$ considered here are phylogenetic, i.e., they satisfy $|\operatorname{child}(v)| \geq 2$ for all $v \in V^{0}(T)$. The last common ancestor of a vertex set $W \subseteq V(T)$ is the unique $\preceq_{T}$-minimal vertex $\operatorname{lca}_{T}(W) \in V(T)$ satisfying $w \preceq_{T} \operatorname{lca}_{T}(W)$ for all $w \in W$. For brevity, we write $\operatorname{lca}_{T}(x, y):=\operatorname{lca}_{T}(\{x, y\})$. Furthermore, we will sometimes write $v u \in E(T)$ as a shorthand for " $\{u, v\} \in E(T)$ with $u \prec_{T} v$." We denote by $T(u)$ the subtree of $T$ rooted in $u$ and write $L(T(u))$ for its leaf set.

Furthermore, $L_{v}^{T}:=\left\{(x, y) \mid x, y \in L(T), \operatorname{lca}_{T}(x, y)=v\right\}$ denotes the set of pairs of leaves that have $v$ as their last common ancestor. By construction, $L_{v}^{T} \cap L_{v^{\prime}}^{T}=\emptyset$ if $v \neq v^{\prime}$. Since $T$ is phylogenetic, we have $L_{v}^{T} \neq \emptyset$ for all $v \in V^{0}(T)$, i.e., $\mathcal{L}(T):=\left\{L_{v}^{T} \mid v \in V^{0}(T)\right\}$ is a partition of the set of distinct pairs of vertices.

A hierarchy on $L$ is set system $\mathcal{H} \subseteq 2^{L}$ such that (i) $L \in \mathcal{H}$, (ii) $A \cap B \in\{A, B, \emptyset\}$ for all $A, B \in \mathcal{H}$, and (iii) $\{x\} \in \mathcal{H}$ for all $x \in L$. There is a well-known bijection between rooted phylogenetic trees $T$ with leaf set $L$ and hierarchies on $L$, see e.g. [19, Thm. 3.5.2]. It is given by $\mathcal{H}(T):=\{L(T(u)) \mid u \in V(T)\}$; conversely, the tree $T_{\mathcal{H}}$ corresponding to a hierarchy $\mathcal{H}$ is the Hasse diagram w.r.t. set inclusion. Thus, if $v=\operatorname{lca}_{T}(A)$ for some $A \subseteq L(T)$, then $L(T(v))$ is the inclusion-minimal cluster in $\mathcal{H}(T)$ that contains $A$ [11].

Let $T$ and $T^{*}$ be phylogenetic trees with $L(T)=L\left(T^{*}\right)$. We say that $T^{*}$ is a refinement of $T$ if $T$ can be obtained from $T^{*}$ by contracting a subset of inner edges or equivalently if and only if $\mathcal{H}(T) \subseteq \mathcal{H}\left(T^{*}\right)$.

Lemma 1. Let $T^{*}$ be a refinement of $T$ and $u^{*} v^{*} \in E\left(T^{*}\right)$. Then there is a unique vertex $w \in V(T)$ such that $L(T(w)) \in \mathcal{H}(T)$ is inclusion-minimal in $\mathcal{H}(T)$ with the property that $L\left(T^{*}\left(v^{*}\right)\right) \subsetneq$ $L(T(w))$. In particular, if $\operatorname{lca}_{T^{*}}(x, y)=u^{*}$, then $\operatorname{lca}_{T}(x, y)=w$.

Proof. Let $u^{*} v^{*} \in E\left(T^{*}\right)$. Since $\mathcal{H}(T) \subseteq \mathcal{H}\left(T^{*}\right), L(T)=L\left(T^{*}\right) \in \mathcal{H}(T)$ and $v^{*}$ is not the root of $T^{*}$, there is a unique inclusion-minimal $A \in \mathcal{H}(T)$ with $L\left(T^{*}\left(v^{*}\right)\right) \subsetneq A$, which corresponds to a unique vertex $w \in V(T)$ that satisfies $L(T(w))=A$. In the following, we denote with $w^{*} \in V\left(T^{*}\right)$ the unique vertex that satisfies $A=L\left(T^{*}\left(w^{*}\right)\right)$, which exists since $A \in \mathcal{H}(T) \subseteq \mathcal{H}\left(T^{*}\right)$. Now let $x, y \in L(T)$ be two leaves with lca $T_{T^{*}}(x, y)=u^{*}$. From $v^{*} \prec_{T^{*}} u^{*}$, we obtain $L\left(T^{*}\left(v^{*}\right)\right) \subsetneq L\left(T^{*}\left(u^{*}\right)\right)$ and $L\left(T^{*}\left(u^{*}\right)\right) \subseteq L\left(T^{*}\left(w^{*}\right)\right)=L(T(w))$. Hence, we have $L\left(T^{*}\left(u^{*}\right)\right) \subseteq L(T(w))$, which implies $x, y \in L(T(w))$ and thus also $z:=\operatorname{lca}_{T}(x, y) \preceq_{T} w$. Denote by $z^{*} \in V\left(T^{*}\right)$ the unique vertex in $T^{*}$ with $L\left(T^{*}\left(z^{*}\right)\right)=L(T(z))$. Since $z \preceq_{T} w$, it satisfies $L\left(T^{*}\left(z^{*}\right)\right) \subseteq L\left(T^{*}\left(w^{*}\right)\right)$. Since $x, y \in L\left(T^{*}\left(z^{*}\right)\right) \cap L\left(T^{*}\left(u^{*}\right)\right) \neq \emptyset$, we either have $L\left(T^{*}\left(u^{*}\right)\right) \subseteq L\left(T^{*}\left(z^{*}\right)\right)$ or $L\left(T^{*}\left(z^{*}\right)\right) \subsetneq L\left(T^{*}\left(u^{*}\right)\right)$. In the second case, we obtain lca $T^{*}(x, y) \preceq_{T^{*}} z^{*} \prec_{T^{*}} u^{*}$, a contradiction to lca $T^{*}(x, y)=u^{*}$. In the first case, we have $L\left(T^{*}\left(v^{*}\right)\right) \subsetneq L\left(T^{*}\left(u^{*}\right)\right) \subseteq L(T(z)) \subseteq L(T(w))$. Due to inclusion minimality of $L(T(w))$ we have $L(T(z))=L(T(w))$. Thus $\operatorname{lca}_{T}(x, y)=z=w$.

Lemma 1 ensures that, for every $u^{*} \in V^{0}\left(T^{*}\right)$, there is a unique $w \in V(T)$ such that $\operatorname{lca}_{T}(x, y)=$ $w$ for all $(x, y) \in L_{u^{*}}^{T^{*}}$, and thus $L_{u^{*}}^{T^{*}} \subseteq L_{w}^{T}$. Thus we have

Corollary 1. If $T^{*}$ is a refinement of $T$, then the partition $\mathcal{L}\left(T^{*}\right)$ is a refinement of $\mathcal{L}(T)$.

\subsection{Symbolic Ultrametrics}

We write $L^{(2)}:=\{(x, y) \mid x, y \in L, x \neq y\}$ for the "off-diagonal" pairs of leafs and let $M$ be a finite set. 
Definition 1. A tree $T$ with leaf set $L$ and labeling $t: V^{0}(T) \rightarrow M$ of its inner vertices explains a map $\delta: L^{(2)} \rightarrow M$ if $t(\operatorname{lca}(x, y))=\delta(x, y)$ for all distinct $x, y \in L$.

Such a map must be symmetric since $\operatorname{lca}_{T}(x, y)=\operatorname{lca}_{T}(y, x)$ for all $x, y \in L$. A shown in [2], a map $\delta: L^{(2)} \rightarrow M$ can be explained by a labeled tree $(T, t)$ if and only if $\delta$ is a symbolic ultrametric, i.e., iff, for all pairwise distinct $u, v, x, y \in L$ holds (i) $\delta(x, y)=\delta(y, x)$ (symmetry), (ii) $\delta(x, y)=\delta(y, u)=\delta(u, v) \neq \delta(y, v)=\delta(x, v)=\delta(x, u)$ is never satisfied (co-graph property), and (iii) $|\{\delta(u, v), \delta(u, x), \delta(v, x)\}| \leq 2$ (exclusion of rainbow triangles). In this case, there exists a unique least-resolved tree $\left(T_{\delta}, t_{\delta}\right)$ (that explains $\delta$ ) with a discriminating vertex labeling $t_{\delta}$, i.e., $t_{\delta}(x) \neq t_{\delta}(y)$ for all $x y \in E\left(T_{\delta}\right)[2,9]$. This tree $\left(T_{\delta}, t_{\delta}\right)$ is also called a discriminating representation of $\delta$ [2].

The construction of symbolic ultrametrics could also be extended to maps $\tilde{\delta}: L^{(2)} \rightarrow 2^{M}$, i.e, to allow multiple labels at each vertex. However, this does not introduce anything new. To see this, we note that the sets of vertex pairs $L_{v}^{T}$ that share the same last common ancestor are pairwise disjoint. In particular, $\tilde{\delta}$ thus must be a fixed element in $2^{M}$ on each $L_{v}^{T}, v \in V^{0}$, and thus we think of the images $\tilde{\delta}(x, y)$ simply as single labels "associated to" elements in $2^{M}$ rather than sets of labels.

Lemma 2. Let $\delta: L^{(2)} \rightarrow M$ be a symbolic ultrametric with least-resolved tree $\left(T_{\delta}, t_{\delta}\right)$. Then there is a map $t: V(T) \rightarrow M$ such that $(T, t)$ explains $\delta$ if and only if $T$ is a refinement of $T_{\delta}$. In this case, the map $t$ is uniquely determined by $T$ and $\delta$.

Proof. Suppose $(T, t)$ explains $\delta$ and let $e=v u \in E(T)$ be an edge with $\delta(u)=\delta(v)$ and $u \prec v$. Note that both $u$ and $v$ must be inner vertices. Let $T / e$ denote the tree obtained from $T$ by contracting the edge $e$, i.e., removing $e$ from $T$ and identifying $u$ and $v$. We will keep the vertex $v$ in $T / e$ as placeholder for the identified vertices $u$ and $v$. By construction, $T / e$ has the clusters $\mathcal{H}(T / e)=\mathcal{H}(T) \backslash\{L(T(u))\}$. Set $t_{T / e}(x)=t(x)$ for all $x \in V^{0}(T) \backslash\{u\}$. Clearly, $v$ is the unique vertex in $T / e$ such that $L((T / e)(v))$ is inclusion-minimal with property $L\left(T\left(u^{\prime}\right)\right) \subsetneq L((T / e)(v))$ for any $u^{\prime} \operatorname{child}_{T}(u)$. Therefore, by Lemma $1, \operatorname{lca}_{T}(x, y)=u \operatorname{implies} \operatorname{lca}_{T / e}(x, y)=v$, and thus, we have $t\left(\operatorname{lca}_{T}(x, y)\right)=t_{T / e}\left(\operatorname{lca}_{T / e}(x, y)\right)$ for all $(x, y) \in L^{(2)}$, and thus $\left(T / e, t_{T / e}\right)$ explains $\delta$. Stepwise contraction of all edges whose endpoints have the same label eventually results in a tree $T^{\prime}$ and a map $t^{\prime}$ such that $t^{\prime}(x) \neq t^{\prime}(y)$ for all edges of $T^{\prime}$. Thus $\left(T^{\prime}, t^{\prime}\right)$ coincides with the unique discriminating representation of $\delta$, i.e., $\left(T^{\prime}, t^{\prime}\right)=\left(T_{\delta}, t_{\delta}\right)$. By construction, $T$ is a refinement of $T_{\delta}$.

Conversely, let $\delta$ be a symbolic ultrametric with (unique) discriminating representation $\left(T_{\delta}, t_{\delta}\right)$ and let $T$ be a refinement of $T_{\delta}$. By Cor. $1, \mathcal{L}(T)$ is a refinement $\mathcal{L}\left(T_{\delta}\right)$. Hence, the map $t$ : $V^{0}(T) \rightarrow M$ specified by $t\left(\operatorname{lca}_{T}(x, y)\right):=t_{\delta}\left(\operatorname{lca}_{T_{\delta}}(x, y)\right)$ for all $(x, y) \in L^{(2)}$ is well-defined. By construction, therefore, $(T, t)$ explains $\delta$. In particular, therefore, every refinement $T$ of $T_{\delta}$ admits a vertex labeling $t$ such that $(T, t)$ explains $\delta$. The choice of $t$ is unique since every inner vertex of a phylogenetic tree is the last common ancestor of at least one pair of vertices, and thus no relabeling of an inner vertex preserves the property that the resulting tree explains $\delta$.

\section{$2.3 \quad$ Fitch Maps}

Definition 2. A tree $T$ with edge labeling $\lambda: E(T) \rightarrow 2^{N}$, with finite $N$, explains a map $\varepsilon: L^{(2)} \rightarrow$ $2^{N}$ if for all $k \in N$ holds: $k \in \varepsilon(x, y)$ iff $k \in \lambda(e)$ for some edge along the unique path in $T$ that connects $\operatorname{lca}_{T}(x, y)$ and $y$.

A map $\varepsilon: L^{(2)} \rightarrow 2^{N}$ that is explained by a tree $(T, \lambda)$ in this manner is a Fitch map [12]. A Fitch map is called monochromatic if $|N|=1$. Like symbolic ultrametrics, Fitch maps are explained by unique least resolved trees. The key construction is provided by the sets $U_{\neg m}[y]:=\{x \in L \backslash\{y\} \mid$ $m \notin \varepsilon(x, y)\} \cup\{y\}$ for $y \in L$ and $m \in N$. Let us write $\mathcal{N}_{\varepsilon}:=\left\{U_{\neg m}[y] \mid y \in L, m \in N\right\}$. Then $\varepsilon$ is a Fitch map if and only if (i) $\mathcal{N}_{\varepsilon}$ is hierarchy-like, i.e., $A \cap B \in\{A, B, \emptyset\}$ for all $A, B \in \mathcal{N}_{\varepsilon}$ and (ii) $\left|U_{\neg m}\left[y^{\prime}\right]\right| \leq\left|U_{\neg m}[y]\right|$ for all $y \in L, m \in N$, and $y^{\prime} \in U_{\neg m}[y][12$, Thm. 3.11].

Fitch maps allow some freedom in distributing labels on the edge set. The precise notion of "least-resolved" thus refers to the fact that it is neither possible to contract edges nor to remove subsets of labels from an edge. The unique least-resolved tree for a Fitch map $\varepsilon$, called the $\varepsilon$-tree $\left(T_{\varepsilon}, \lambda_{\varepsilon}\right)$, is determined by the hierarchy $\mathcal{H}\left(T_{\varepsilon}\right)=\mathcal{N}_{\varepsilon} \cup\{L\} \cup\{\{x\} \mid x \in L\}$ and the labeling $\lambda_{\varepsilon}(\operatorname{parent}(v), v):=\left\{m \in N \mid \exists y \in L\right.$ s.t. $\left.L\left(T_{\varepsilon}(v)\right)=U_{\neg m}[y]\right\}$ for all $e=\{\operatorname{parent}(v), v\} \in E\left(T_{\varepsilon}\right)$ [12, Thm. 4.4].

Let $(T, \lambda)$ and $\left(T^{\prime}, \lambda^{\prime}\right)$ be two edge-labeled trees on the same leaf set and with $\lambda: E(T) \rightarrow 2^{N}$ and $\lambda^{\prime}: E\left(T^{\prime}\right) \rightarrow 2^{N}$. Then $(T, \lambda)$ is a refinement of $\left(T^{\prime}, \lambda^{\prime}\right)$, in symbols $\left(T^{\prime}, \lambda^{\prime}\right) \leq(T, \lambda)$ if (i) $\mathcal{H}\left(T^{\prime}\right) \subseteq \mathcal{H}(T)$ and (ii) if $L(T(v))=L\left(T^{\prime}\left(v^{\prime}\right)\right)$, then $\lambda^{\prime}\left(\operatorname{parent}_{T^{\prime}}\left(v^{\prime}\right), v^{\prime}\right) \subseteq \lambda\left(\right.$ parent $\left._{T}(v), v\right)$. 
Proposition 1. [12, Prop.4.3, Thm.4.4] If $(T, \lambda)$ explains $\varepsilon$, then $\left(T_{\varepsilon}, \lambda_{\varepsilon}\right) \leq(T, \lambda)$. Furthermore, $\left(T_{\varepsilon}, \lambda_{\varepsilon}\right)$ is the unique least-resolved tree that explains $\varepsilon$. In particular, $\left(T_{\varepsilon}, \lambda_{\varepsilon}\right)$ minimizes $\ell_{\min }:=$ $\sum_{e \in E\left(T_{\varepsilon}\right)}\left|\lambda_{\varepsilon}(e)\right|$.

Lemma 3. Let $\varepsilon: L^{(2)} \rightarrow 2^{N}$ be a Fitch map with least-resolved tree $\left(T_{\varepsilon}, \lambda_{\varepsilon}\right)$. Then there exists an edge labeling $\lambda: E(T) \rightarrow 2^{N}$ such that $(T, \lambda)$ explains $\varepsilon$ if and only if $T$ is a refinement of $T_{\varepsilon}$.

Proof. Suppose $(T, \lambda)$ explains $\varepsilon$. By Prop. 1, this implies $\left(T_{\varepsilon}, \lambda_{\varepsilon}\right) \leq(T, \lambda)$, i.e., $T$ is a refinement of $T_{\varepsilon}$. Conversely, let $\varepsilon$ be a Fitch map with least-resolved tree $\left(T_{\varepsilon}, \lambda_{\varepsilon}\right)$ and let $T$ be a refinement of $T_{\varepsilon}$. Define, for all edges $\left\{\operatorname{parent}_{T}(v), v\right\} \in E(T)$, the edge labeling

$$
\lambda\left(\left\{\operatorname{parent}_{T}(v), v\right\}\right):= \begin{cases}\lambda_{\varepsilon}\left(\operatorname{parent}_{T_{\varepsilon}}\left(v^{\prime}\right), v^{\prime}\right) & \text { if } L(T(v))=L\left(T_{\varepsilon}\left(v^{\prime}\right)\right), \\ \emptyset & \text { otherwise. }\end{cases}
$$

The map $\lambda$ is well-defined, since there is at most one $v^{\prime} \in V\left(T_{\varepsilon}\right)$ with $L(T(v))=L\left(T_{\varepsilon}\left(v^{\prime}\right)\right)$.

Claim. $(T, \lambda)$ and $\left(T_{\varepsilon}, \lambda_{\varepsilon}\right)$ explain the same Fitch map $\varepsilon$.

By assumption, $\left(T_{\varepsilon}, \lambda_{\varepsilon}\right)$ explains $\varepsilon$. Let $(a, b) \in L^{(2)}, k \in N$, and let $\varepsilon^{\prime}$ be the Fitch map explained by $(T, \lambda)$. First, suppose $k \in \varepsilon(a, b)$, i.e., there is an edge $e^{\prime}=\left\{\right.$ parent $\left._{T_{\varepsilon}}\left(w^{\prime}\right), w^{\prime}\right\}$ with $k \in \lambda_{\varepsilon}\left(e^{\prime}\right)$ such that $w^{\prime} \prec_{T_{\varepsilon}} \operatorname{lca}_{T_{\varepsilon}}(a, b)$ by the definition of Fitch maps. We have $a \notin L\left(T_{\varepsilon}\left(w^{\prime}\right)\right)$. Since $T$ is a refinement of $T_{\varepsilon}$, there is a vertex $w \in V(T)$ with $L(T(w))=L\left(T_{\varepsilon}\left(w^{\prime}\right)\right)$. In particular, therefore, $\lambda\left(\left\{\right.\right.$ parent $\left.\left._{T}(w), w\right\}\right)=\lambda_{\varepsilon}\left(e^{\prime}\right)$. This together with the fact that $a \notin L\left(T_{\varepsilon}\left(w^{\prime}\right)\right)=L(T(w))$ immediately implies $k \in \varepsilon^{\prime}(a, b)$. Now suppose $k \in \varepsilon^{\prime}(a, b)$. Hence, there is an edge $e=\left\{\operatorname{parent}_{T}(v), v\right\}$ with $v \prec_{T}$ lca $(a, b)$ and $k \in \lambda(e)$. By construction of $\lambda$, the latter implies that there is a vertex $v^{\prime} \in V\left(T_{\varepsilon}\right)$ with $L(T(v))=L\left(T_{\varepsilon}\left(v^{\prime}\right)\right)$ and, in particular, $k \in \lambda_{\varepsilon}$ parent $\left._{T_{\varepsilon}}\left(v^{\prime}\right), v^{\prime}\right)$. The latter together with $a \notin L(T(v))=L\left(T_{\varepsilon}\left(v^{\prime}\right)\right)$ implies that $k \in \varepsilon(a, b)$. Since $(a, b) \in L^{(2)}$ and $k \in N$ were chosen arbitrarily, we conclude that $\varepsilon=\varepsilon^{\prime}$, and thus, $(T, \lambda)$ also explains $\varepsilon$.

The labeling $\lambda$ defined in Eq.(1) satisfies $\ell_{\min }=\sum_{e \in T(e)}|\lambda(e)|$ by construction and Prop. 1. Furthermore, we observe that $\left(T^{*}, \lambda^{*}\right)$ is obtained from $(T, \lambda)$ by contracting only edges with $\lambda(e)=\emptyset$. More precisely, $e$ is contracted if and only if $e$ is an inner edge with $\lambda(e)=\emptyset$. This implies

Corollary 2. Suppose $\left(T, \lambda^{\prime}\right)$ explains the Fitch map $\varepsilon$. Then $\lambda: E(T) \rightarrow 2^{N}$ given by Eq. (1) is the unique labeling such that $(T, \lambda)$ explains $\varepsilon$ and $\sum_{e \in E(T)}|\lambda(e)|=\ell_{\min }$.

Proof. Suppose $\left(T, \lambda^{\prime \prime}\right)$ explains $\varepsilon$ and $\sum_{e \in E(T)}\left|\lambda^{\prime \prime}(e)\right|=\ell_{\min }$. By Prop. 1, we have $\left(T_{\varepsilon}, \lambda_{\varepsilon}\right) \leq$ $\left(T, \lambda^{\prime \prime}\right)$ and thus $\lambda_{\varepsilon}\left(\right.$ parent $\left._{T_{\varepsilon}}\left(v^{\prime}\right), v^{\prime}\right) \subseteq \lambda^{\prime \prime}\left(\right.$ parent $\left._{T}(v), v\right)$ if $L\left(T_{\varepsilon}\left(v^{\prime}\right)\right)=L(T(v))$. Since, moreover, $\lambda_{\varepsilon}\left(\operatorname{parent}_{T_{\varepsilon}}\left(v^{\prime}\right), v^{\prime}\right)=\lambda\left(\right.$ parent $\left._{T}(v), v\right)$ if $L\left(T_{\varepsilon}\left(v^{\prime}\right)\right)=L(T(v))$ by Eq.(1), minimality of $\lambda^{\prime \prime}$ implies $\lambda^{\prime \prime}=\lambda$.

\section{Tree-like Pairs of Maps}

Symbolic ultrametrics and Fitch maps on $L^{(2)}$ derive from trees in very different ways by implicitly leveraging information about inner vertices and edges of the a priori unknown tree. It is of interest, therefore, to know when they are consistent in the sense that they can be simultaneously explained by a tree.

Definition 3. An ordered pair $(\delta, \varepsilon)$ of maps $\delta: L^{(2)} \rightarrow M$ and $\varepsilon: L^{(2)} \rightarrow 2^{N}$ is tree-like if there is a tree $T$ endowed with a vertex labeling $t: V^{0}(T) \rightarrow M$ and edge labeling $\lambda: L^{(2)} \rightarrow 2^{N}$ such that $(T, t)$ explains $\delta$ and $(T, \lambda)$ explains $\varepsilon$.

Naturally, we ask when $(\delta, \varepsilon)$ is explained by a vertex and edge labeled tree $(T, t, \lambda)$, i.e., when $(\delta, \varepsilon)$ is a tree-like pair of maps on $L^{(2)}$. Furthermore, we ask whether a tree-like pair of maps is again explained by a unique least-resolved tree $\left(T^{*}, t^{*}, \lambda^{*}\right)$.

Theorem 1. Let $\delta: L^{(2)} \rightarrow M$ and $\varepsilon: L^{(2)} \rightarrow 2^{N}$. Then $(\delta, \varepsilon)$ is tree-like if and only if

1. $\delta$ is a symbolic ultrametric.

2. $\varepsilon$ is a Fitch map.

3. $\mathcal{H}^{*}:=\mathcal{H}\left(T_{\delta}\right) \cup \mathcal{H}\left(T_{\varepsilon}\right)$ is a hierarchy.

In this case, there is a unique least-resolved vertex and edge labeled tree $\left(T^{*}, t^{*}, \lambda^{*}\right)$ explaining $(\delta, \varepsilon)$. 
The tree $T^{*}$ is determined by $\mathcal{H}\left(T^{*}\right)=\mathcal{H}^{*}$, the vertex labeling $t^{*}$ is uniquely determined by $t_{\delta}$ and the edge labeling $\lambda^{*}$ with minimum value of $\sum_{e \in E\left(T^{*}\right)}\left|\lambda^{*}(e)\right|$ is uniquely determined by $\lambda_{\varepsilon}$.

Proof. Suppose $(\delta, \varepsilon)$ is tree-like, i.e., there is a tree $(T, t, \lambda)$ such that $(T, t)$ explains $\delta$ and $(T, \lambda)$ explains $\varepsilon$. Thus $\delta$ is a symbolic ultrametric and $\varepsilon$ is a Fitch map. Furthermore, $T$ is a refinement of least-resolved trees $T_{\delta}$ and $T_{\varepsilon}$ because of the uniqueness of these least-resolved trees, and we have $\mathcal{H}\left(T_{\delta}\right) \subseteq \mathcal{H}(T)$ and $\mathcal{H}\left(T_{\varepsilon}\right) \subseteq \mathcal{H}(T)$ and thus $\mathcal{H}^{*} \subseteq \mathcal{H}(T)$. Since $\mathcal{H}(T)$ is a hierarchy and the subset $\mathcal{H}^{*}$ contains both $L$ and all singletons $\{x\}$ with $x \in L, \mathcal{H}^{*}$ is a hierarchy.

Conversely, suppose conditions (1), (2), and (3) are satisfied. The first two conditions guarantee the existence of the least-resolved tree $\left(T_{\delta}, t_{\delta}\right)$ and $\left(T_{\varepsilon}, \lambda_{\varepsilon}\right)$ explaining $\delta$ and $\varepsilon$, respectively. Thus $\mathcal{H}^{*}=\mathcal{H}\left(T_{\delta}\right) \cup \mathcal{H}\left(T_{\varepsilon}\right)$ is well-defined. Condition (3) stipulates that $\mathcal{H}^{*}$ is a hierarchy and thus there is a unique tree $T^{*}$ such that $\mathcal{H}\left(T^{*}\right)=H^{*}$, which by construction is a refinement of both $T_{\delta}$ and $T_{\varepsilon}$. By Lemmas 2 and 3, $T^{*}$ can be equipped with a vertex-labeling $t^{*}$ and an edge-labeling $\lambda^{*}$ such that $\left(T^{*}, t^{*}\right)$ explains $\delta$ and $\left(T^{*}, \lambda^{*}\right)$ explains $\varepsilon$, respectively. Thus $(\delta, \varepsilon)$ is tree-like.

We now show that $\left(T^{*}, t^{*}, \lambda^{*}\right)$ is least-resolved w.r.t. $(\delta, \varepsilon)$ and thus that for every $e \in E\left(T^{*}\right)$, the tree $T^{\prime}:=T^{*} / e$ does not admit a vertex labeling $t^{\prime}: V^{0}\left(T^{\prime}\right) \rightarrow M$ and an edge-labeling $\lambda^{\prime}: E\left(T^{\prime}\right) \rightarrow 2^{N}$ such that $\left(T^{\prime}, t^{\prime}, \lambda^{\prime}\right)$ explains $(\delta, \varepsilon)$. Let $e=\{\operatorname{parent}(v), v\} \in E\left(T^{*}\right)$. Hence, $L\left(T^{*}(v)\right) \in \mathcal{H}\left(T^{*}\right)$. If $v \in L\left(T^{*}\right)$, then we have $L\left(T^{*}\right) \neq L\left(T^{\prime}\right)$ and the claim trivially holds. Thus suppose that $v \in V^{0}(T)$ in the following. Since the edge $e$ is contracted in $T^{\prime}$, we have $\mathcal{H}\left(T^{\prime}\right)=\mathcal{H}\left(T^{*}\right) \backslash\{L(T(v))\}$ and thus, $\mathcal{H}\left(T_{\delta}\right) \nsubseteq \mathcal{H}\left(T^{\prime}\right)$ or $\mathcal{H}\left(T_{\varepsilon}\right) \nsubseteq \mathcal{H}\left(T^{\prime}\right)$. Thus $T^{\prime}$ is not a refinement of $T_{\delta}$ or $T_{\varepsilon}$. By Lemma 2 and 3 , respectively, this implies that there is no $t^{\prime}$ such that $\left(T^{\prime}, t^{\prime}\right)$ explains $\delta$ or no $\lambda^{\prime}$ such that $\left(T^{\prime}, \lambda^{\prime}\right)$ explains $\varepsilon$, respectively. Thus $\left(T^{*}, t^{*}, \lambda^{*}\right)$ is least-resolved w.r.t. $(\delta, \varepsilon)$.

It remains to show that $\left(T^{*}, t^{*}, \lambda^{*}\right)$ is unique. Since $T^{*}$ is uniquely determined by $\mathcal{H}^{*}$, it suffices to show that the labeling of $T^{*}$ is unique. This, however, follows immediately from Lemma 2 and Cor. 2, respectively.

We note that every refinement $T$ of the least-resolved tree $\left(T^{*}, t^{*}, \lambda^{*}\right)$ admits a vertex labeling $t: V^{0}(T) \rightarrow M$ and an edge labeling $\lambda: E(T) \rightarrow 2^{N}$ such that $(T, t, \lambda)$ explains $(\delta, \varepsilon)$.

Theorem 2. Given two maps $\delta: L^{(2)} \rightarrow M$ and $\varepsilon: L^{(2)} \rightarrow 2^{N}$ it can be decided in $O\left(|L|^{2}|N|\right)$ whether $(\delta, \varepsilon)$ is tree-like. In the positive case, the unique least-resolved tree $\left(T^{*}, t^{*}, \lambda^{*}\right)$ can be obtained with the same effort.

Proof. Based on Theorem 1, a possible algorithm consists of three steps: (i) check whether $\delta$ is a symbolic ultrametric, (ii) check whether $\varepsilon$ is a Fitch map and, if both statements are true, (iii) compute $\mathcal{H}^{*}:=\mathcal{H}\left(T_{\delta}\right) \cup \mathcal{H}\left(T_{\varepsilon}\right)$ and use this information to compute the unique least-resolved vertex and edge labeled tree $\left(T^{*}, t^{*}, \lambda^{*}\right)$. By [12, Thm. 6.2], the decision whether $\varepsilon$ is a Fitch map and, in the positive case, the construction of the least-resolved tree $\left(T_{\varepsilon}, \lambda_{\varepsilon}\right)$ can be achieved in $O\left(|L|^{2}|N|\right)$ time. Moreover, it can be verified in $O\left(|L|^{2}\right)$ whether or not a given map $\delta$ is a symbolic ultrametric, and, in the positive case, the discriminating tree $\left(T_{\delta}, t_{\delta}\right)$ can be computed within the same time complexity (cf. [14, Thm. 7]). The common refinement $T$ with $\mathcal{H}(T)=\mathcal{H}\left(T_{\delta}\right) \cup \mathcal{H}\left(T_{\varepsilon}\right)$ can be computed in $O(|L|)$ time using LinCR [18].

The edge labels $\lambda^{*}$ are then carried over from $\left(T_{\varepsilon}, \lambda_{\varepsilon}\right)$ using the correspondence between $u^{*} v^{*} \in$ $E\left(T^{*}\right)$ and $u v \in E\left(T_{\varepsilon}\right)$ iff $L\left(T^{*}\left(v^{*}\right)\right)=L\left(T_{\varepsilon}(v)\right)$, otherwise $\lambda^{*}\left(\left\{u^{*}, v^{*}\right\}\right)=\emptyset$. This requires $O(|L| \cdot|N|)$ operations. The vertex labels can then be assigned by computing, for all $(x, y) \in L^{(2)}$, the vertex $v=\operatorname{lca}_{T^{*}}(x, y)$ and assigning $t^{*}(v)=\delta(x, y)$ in quadratic time using a fast last common ancestor algorithm [7]. Thus we arrive at a total performance bounds of $\left.O\left(|L|^{2}|N|\right)\right)$.

\section{Tree-like Pairs of Maps with Constraints}

One interpretation of tree-like pairs of maps $(\delta, \varepsilon)$ is to consider $\delta$ as the orthology relation and $\varepsilon$ as the xenology relation. In such a setting, certain vertex labels $t(v)$ preclude some edge labels $\lambda(\{v, u\})$ with $u \prec v$. For example, a speciation vertex cannot be the source of a horizontal transfer edge. We use the conventional notations $t(u)=\boldsymbol{\bullet}$ and $t(v)=\square$ for speciation and duplication vertices [6], respectively, set $t(u)=\triangle$ for a third vertex type, and consider the monochromatic Fitch map $\varepsilon: L^{(2)} \rightarrow\{\emptyset, \mathbb{I}\}$. Thus, we require that $\lambda(\{v, u\})=\mathbb{I}$ and $u \prec_{T} v$ implies $t(v)=\triangle$ $[17,20,1]$. This condition simply states that neither a speciation nor a gene duplication is the source of a horizontal transfer.

In [17], we considered evolutionary scenarios that satisfy another rather stringent observability condition: 

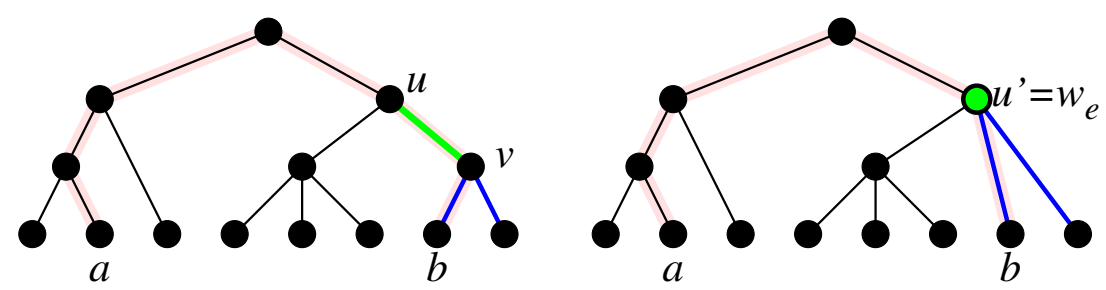

Figure 1: Effect of an edge contraction on paths in $T$. All paths traversing the contracted edge $e=u v$ in $T$ correspond to paths in $T / e$ in which $e$ is contracted. All other path remain unchanged. Furthermore $w_{e}=u^{\prime}$, i.e., the edge contraction corresponds to the deletion of $L(T(v))$ from $\mathcal{H}(T)$.

(C) For every $v \in V^{0}(T)$, there is a child $u \in \operatorname{child}(v)$ such that $\lambda(\{v, u\})=\emptyset$.

We call a Fitch map $\lambda$ that satisfies (C) a type-C Fitch map. In this case, for every $v \in V^{0}(T)$, there is a leaf $x \in L(T(v))$ such that $\lambda(e)=\emptyset$ for all edges along the path from $v$ to $x$. As an immediate consequence of $(\mathrm{C})$, we observe that, given $|L| \geq 2$, for every $x \in L$ there is a $y \neq x$ such that $\varepsilon(x, y)=\emptyset$. This condition is not sufficient, however, as the following example shows. Consider the tree $\left((x, y),\left(x^{\prime}, y^{\prime}\right)\right)$ in Newick notation, with the edges in the two cherries $(x, y)$ and $\left(x^{\prime}, y^{\prime}\right)$ being labeled with $\emptyset$, and two $\mathbb{I}$-labeled edges incident to the root. Then, for every $z \in L$, we have $\varepsilon\left(z, z^{\prime}\right)=\emptyset$, where $z^{\prime}$ is the sibling of $z$, but condition $(\mathrm{C})$ is not satisfied. In a somewhat more general setting, we formalize these two types of labeling constraints as follows:

Definition 4. Let $\delta: L^{(2)} \rightarrow M$ and $\varepsilon: L^{(2)} \rightarrow 2^{N}$ be two maps and $M_{\emptyset} \subseteq M$. Then, $(\delta, \varepsilon)$ is $M_{\emptyset}$-tree-like if there is a tree $(T, t, \lambda)$ that explains $(\delta, \varepsilon)$ and the labeling maps $t: V^{0}(T) \rightarrow M$ and $\lambda: E(T) \rightarrow 2^{N}$ satisfy $(\mathrm{C})$ and

(C1) If $t(v) \in M_{\emptyset}$, then $\lambda(\{v, u\})=\emptyset$ for all $u \in \operatorname{child}(v)$.

Hence, $M_{\emptyset}$ puts extra constraints to the vertex and edge labels on trees that satisfy $(\mathrm{C})$ and explain $(\delta, \varepsilon)$. Note, an $\emptyset$-tree-like $\left(M_{\emptyset}=\emptyset\right)$ must only satisfy $(\mathrm{C})$ and $(\mathrm{C} 1)$ can be omitted.

Theorem 3. Let $\delta: L^{(2)} \rightarrow M$ and $\varepsilon: L^{(2)} \rightarrow 2^{N}$ be two maps and $M_{\emptyset} \subseteq M$. Then, $(\delta, \varepsilon)$ is $M_{\emptyset}$-tree-like if and only if $(\delta, \varepsilon)$ is tree-like and its least-resolved tree $\left(T^{*}, t^{*}, \lambda^{*}\right)$ satisfies $(\mathrm{C})$ and (C1).

Proof. If $(\delta, \varepsilon)$ is tree-like and its least-resolved tree $\left(T^{*}, t^{*}, \lambda^{*}\right)$ satisfies $(\mathrm{C})$ and $(\mathrm{C} 1)$, then $(\delta, \varepsilon)$ is $M_{\emptyset}$-tree-like by definition. For the converse, suppose $(\delta, \varepsilon)$ is $M_{\emptyset}$-tree-like and let $(T, t, \lambda)$ be a vertex and edge labeled tree that explains $(\delta, \varepsilon)$ and satisfies $(\mathrm{C})$ and $(\mathrm{C} 1)$.

Let $\lambda^{\prime}$ be the edge labeling for $T$ as specified in Eq.(1) where $\left(T_{\varepsilon}, \lambda_{\varepsilon}\right)$ is replaced by $\left(T^{*}, \lambda^{*}\right)$. By the arguments in the proof of Lemma $3,\left(T, \lambda^{\prime}\right)$ still explains $\varepsilon$ and hence, $\left(T, t, \lambda^{\prime}\right)$ explains $(\delta, \varepsilon)$. Moreover, since $\ell_{\min }:=\sum_{e \in E\left(T^{*}\right)}\left|\lambda^{*}(e)\right|$ and by construction of $\lambda^{\prime}$, we have $\ell_{\min }=\sum_{e \in E(T)}\left|\lambda^{\prime}(e)\right|$. Since $\left(T^{*}, \lambda^{*}\right) \leq\left(T, \lambda^{\prime}\right)$, it must hold $\lambda_{\varepsilon}\left(e^{\prime}\right) \subseteq \lambda^{\prime}(e)$ for all $e^{\prime}=\operatorname{parent}\left(v^{\prime}\right) v^{\prime} \in E\left(T_{\varepsilon}\right)$ and $e=$ parent $(v) v \in E(T)$ with $L(T(v))=L\left(T_{\varepsilon}\left(v^{\prime}\right)\right)$. Since $\lambda^{\prime}$ is minimal by construction, we have $\lambda_{\varepsilon}\left(e^{\prime}\right)=\lambda^{\prime}(e)$ for all corresponding edges $e$ and $e^{\prime}$. In particular, it must hold that $\lambda(e)=\emptyset$ implies $\lambda^{\prime}(e)=\emptyset$ for all $e \in E(T)$. To see this, assume for contradiction there is some edge $e=u v \in E(T)$ with $\lambda(e)=\emptyset$ but $\lambda^{\prime}(e) \neq \emptyset$. Since $(T, \lambda)$ satisfies $(\mathrm{C})$, there is a path from $u$ to some leaf $y \in L(T)$ that consists of edges $f$ with label $\lambda(f)=\emptyset$ only and that contains the edge $e$. Hence, for $x \in L(T(u)) \backslash L(T(v))$, we have $\operatorname{lca}_{T}(x, y)=u$ and thus, $\varepsilon(x, y)=\emptyset$. However, since we assume that $\lambda^{\prime}(e)=N^{\prime} \neq \emptyset$, we obtain $N^{\prime} \subseteq \varepsilon(x, y) \neq \emptyset$; a contradiction. Now it is easy to verify that $\left(T, t, \lambda^{\prime}\right)$ still satisfies $(\mathrm{C})$ and $(\mathrm{C} 1)$.

Now consider edge contractions, Fig. 1. To obtain $T^{*}$ we are only allowed to contract edges $e=u v \in E(T)$ that satisfy $t(u)=t(v)$ and $\lambda^{\prime}(e)=\emptyset$. The latter follows from the fact that edges $u v$ with $t(u) \neq t(v)$ cannot be contracted without losing the information of at least one of the labels $t(u)$ or $t(v)$ and minimality of $\lambda^{\prime}$, since otherwise the labels $\lambda^{\prime}(e)$ do not contribute to the explanation of the Fitch map and thus would have been removed in the construction of $\lambda^{\prime}$. For such an edge $e$, the tree $\left(T / e, t_{T / e}, \lambda_{T / e}^{\prime}\right)$ is obtained by contracting the edge $e=u v$ to a new vertex $w_{e}$ and assigning $t_{T / e}\left(w_{e}\right)=t(v)=t(u)$ and keeping the edge labels of all remaining edges. The tree $\left(T / e, t_{T / e}, \lambda_{T / e}^{\prime}\right)$ then explains $(\delta, \varepsilon)$. To see this, we write $y:=\operatorname{lca}_{T}(a, b)$ and $y^{\prime}:=\operatorname{lca}_{T / e}(a, b)$ for distinct $a, b \in L$ and compare for $c \in\{a, b\}$ the path $P_{y c}$ in $T$ and $P_{y^{\prime} c}^{\prime}$ in $T / e$. If $y=u$ or $y=v$ then $y^{\prime}=w_{e}$. The paths therefore either consist only of corresponding edges, in which case the edge labels are the same, or they differ exactly by the contraction of $e$. The latter does not 
affect the explanation of $\varepsilon(a, b)$ because $\lambda^{\prime}(e)=\emptyset$. Since $t(u)=t(v)$, contraction of $u v$ also does not affect $\delta$.

In particular, therefore, neither $u$ nor $v$ is a leaf, i.e., $e$ is an inner edge. Condition $(\mathrm{C})$ is trivially preserved under contraction of inner edges. Suppose $t(v)=t(u) \in M_{\emptyset}$ and thus $t_{T / e}\left(w_{e}\right) \in M_{\emptyset}$. Since $\left(T, t, \lambda^{\prime}\right)$ satisfies (C1) we have $\lambda^{\prime}\left(\left\{v, u^{\prime}\right\}\right)=\lambda^{\prime}\left(\left\{u, u^{\prime \prime}\right\}\right)=\emptyset$ for all $u^{\prime} \in \operatorname{child}(v)$ and all $u^{\prime \prime} \in \operatorname{child}(u)$ and thus after contracting $e$ it holds that $\lambda_{T / e}^{\prime}\left(w_{e}, w^{\prime}\right)=\emptyset$ for all $w^{\prime} \in \operatorname{child}_{T / e}\left(w_{e}\right)=$ $\operatorname{child}_{T}(v) \bullet \operatorname{child}_{T}(u)$. Otherwise, $t(u)=t(v) \notin M_{\emptyset}$ and thus by construction $t_{T / e}\left(w_{e}\right) \notin M_{\emptyset}$. In summary, $\left(T / e, t^{\prime}, \lambda\right)$ satisfies $(\mathrm{C})$ and $(\mathrm{C} 1)$. Repeating this coarse graining until no further contractible inner edges are available results in the unique least-resolved tree $\left(T^{*}, t^{*}, \lambda^{*}\right)$.

Since the unique least-resolved tree $\left(T^{*}, t^{*}, \lambda^{*}\right)$ can be computed in quadratic time by Thm. 2, and it suffices by Thm. 3 to check $(\mathrm{C})$ and $(\mathrm{C} 1)$ for $\left(T^{*}, t^{*}, \lambda^{*}\right)$, the same performance bound applies to the recognition of constrained tree-like pairs of maps.

We note that an analogous result holds if only $(\mathrm{C})$ or only $(\mathrm{C} 1)$ is required for $(T, t, \lambda)$. Furthermore, one can extend (C1) in such a way that for a set $\mathcal{Q}$ of pairs $(q, m)$ with $q \in M$ and $m \in N$ of labels that are incompatible at a vertex $v$ and an edge $v v^{\prime}$ with $v^{\prime} \in \operatorname{child}(v)$. The proof of Thm. 3 still remains valid since also in this case no forbidden combinations of vertex an edge colors can arise from contracting an edge $e=u v$ with $t(u)=t(v)$. In the special case $\delta(x, y)=1 \notin M_{\emptyset}$ for all $(x, y) \in L^{(2)}$, one obtains $t^{*}(u)=1$ for all $u \in V\left(T^{*}\right)$ and thus $\left(T^{*}, \lambda^{*}\right)=\left(T_{\varepsilon}, \lambda_{\varepsilon}\right)$ and $(\mathrm{C} 1)$ imposes no constraint. Hence, Thm. 3 specializes to

Corollary 3. A Fitch map $\varepsilon$ is type- $C$ if and only if its least-resolved tree $\left(T_{\varepsilon}, \lambda_{\varepsilon}\right)$ satisfies $(\mathrm{C})$.

In [17] a stronger version of condition $(\mathrm{C})$ has been considered:

(C2) If $\lambda(\{v, u\}) \neq \emptyset$ for some $u \in \operatorname{child}(v)$, then $\lambda\left(\left\{v, u^{\prime}\right\}\right)=\emptyset$ for all $u^{\prime} \in \operatorname{child}(v) \backslash\{u\}$.

This variant imposes an additional condition on the edges $e=u v$ that can be contracted. More precisely, an inner edge of $(T, t, \lambda)$ can be contracted without losing the explanation of $(\delta, \varepsilon)$ and properties (C1) and (C2) if and only if (i) $t(u)=t(v)$, (ii) $\lambda(e)=\emptyset$ and (iii) at most one of the the edges $u u^{\prime}, u^{\prime} \in \operatorname{child}(u)$ and $v v^{\prime}, v^{\prime} \in \operatorname{child}(v)$ has a non-empty label. Now consider two consecutive edges $u v$ and $v w$ with $t(u)=t(v)=t(w), \lambda(\{u, v\})=\lambda(\{v, w\})=\emptyset$ and suppose there is $u^{\prime} \operatorname{child}(u)$ with $\lambda\left(\left\{u, u^{\prime}\right\}\right) \neq \emptyset, w^{\prime} \in \operatorname{child}(w)$ with $\lambda\left(\left\{w, w^{\prime}\right\}\right) \neq \emptyset$, and $\lambda\left(\left\{v, v^{\prime}\right\}\right)=\emptyset$ for all $v^{\prime} \in \operatorname{child}(v)$. Then one can contract either $u v$ or $v w$ but not both edges. Thus least-resolved trees explaining $(\delta, \varepsilon)$ and satisfying $(\mathrm{C} 1)$ and $(\mathrm{C} 2)$ are no longer unique.

\section{Concluding Remark}

Here we have shown that symbolic ultrametrics and Fitch maps can be combined by the simple and easily verified condition that $\mathcal{H}\left(T_{\delta}\right) \cup \mathcal{H}\left(T_{\varepsilon}\right)$ is again a hierarchy (Thm. 1), i.e., that the two leastresolved trees have a common refinement. The least-resolved tree $\left(T^{*}, t^{*}, \lambda^{*}\right)$ that simultaneously explains both $\delta$ and $\varepsilon$ is unique in this case and can be computed in quadratic time if the label set $N$ is bounded and $O\left(|L|^{2}|N|\right)$ time in general. The closely related problem of combining a hierarchy and symmetrized Fitch maps, defined by $m \in \varepsilon(x, y)$ iff there is an edge $e$ with $m \in \lambda(e)$ along the path from $x$ to $y$ [10], is NP-complete [13]. It appears that the main difference is the fact that symmetrized Fitch maps do not have a unique least-resolved tree as explanation. The distinction between much simpler problems in the directed setting and hard problems in the undirected case is also reminiscent of the reconciliation problem for trees, which are easy for rooted trees and hard for unrooted trees, see e.g. [3].

We have also seen that certain restrictions on the Fitch maps that are related to the "observability" of horizontal transfer do not alter the complexity of the problem. These observability conditions are defined in terms of properties of the explaining trees, raising the question whether these constraints also have a natural characterization as properties of the Fitch maps. On a more general level, both symbolic ultrametrics and Fitch maps arise from evolutionary scenarios comprising an embedding of the gene tree $T$ into a species tree, with labeling functions $t$ and $\lambda$ on $T$ encoding event-types and distinctions in the evolutionary fate of offsprings, respectively. Here we have focused entirely on gene trees with given labels. The embeddings into species trees are known to impose additional constraints $[8,16]$.

Acknowledgments. This work was supported in part by the Deutsche Forschungsgemeinschaft. 


\section{References}

[1] M. Bansal, E. Alm, and M. Kellis. Efficient algorithms for the reconciliation problem with gene duplication, horizontal transfer and loss. Bioinformatics, 28:i283-i291, 2012.

[2] S. Böcker and A. Dress. Recovering symbolically dated, rooted trees from symbolic ultrametrics. Adv. Math., 138:105-125, 1998.

[3] D. Bryant and J. Lagergren. Compatibility of unrooted phylogenetic trees is FPT. Theor. Comp. Sci., 351:296-302, 2006.

[4] W. Fitch. Homology: a personal view on some of the problems. Trends Genet., 16:227-231, 2000.

[5] M. Geiß, J. Anders, P. Stadler, N. Wieseke, and M. Hellmuth. Reconstructing gene trees from Fitch's xenology relation. J. Math. Biol., 77:1459-1491, 2018.

[6] M. Geiß, M. González Laffitte, A. López Sánchez, D. Valdivia, M. Hellmuth, M. Hernández Rosales, and P. Stadler. Best match graphs and reconciliation of gene trees with species trees. J. Math. Biol., 80:1459-1495, 2020.

[7] D. Harel and R. Tarjan. Fast algorithms for finding nearest common ancestors. SIAM J. Computing, 13:338-355, 1984.

[8] M. Hellmuth. Biologically feasible gene trees, reconciliation maps and informative triples. Alg. Mol. Biol., 12:23, 2017.

[9] M. Hellmuth, M. Hernandez-Rosales, K. Huber, V. Moulton, P. Stadler, and N. Wieseke. Orthology relations, symbolic ultrametrics, and cographs. J. Math. Biol., 66:399-420, 2013.

[10] M. Hellmuth, Y. Long, M. Geiß, and P. Stadler. A short note on undirected fitch graphs. Art Discr. Appl. Math., 1:P1.08, 2018.

[11] M. Hellmuth, D. Schaller, and P. Stadler. Compatibility of partitions, hierarchies, and split systems. 2021. submitted; arXiv 2104.14146.

[12] M. Hellmuth, C. Seemann, and P. Stadler. Generalized fitch graphs II: Sets of binary relations that are explained by edge-labeled trees. Discr. Appl. Math., 283:495-511, 2020.

[13] M. Hellmuth, C. R. Seemann, and P. F. Stadler. Generalized Fitch graphs III: Symmetrized Fitch maps and sets of symmetric binary relations that are explained by unrooted edge-labeled trees. Discr. Math. Theor. Comp. Sci, 23(1):13, 2021.

[14] M. Hellmuth, P. Stadler, and N. Wieseke. The mathematics of xenology: Di-cographs, symbolic ultrametrics, 2-structures and tree-representable systems of binary relations. J. Math. Biol., 75:199-237, 2017.

[15] M. Jones, M. Lafond, and C. Scornavacca. Consistency of orthology and paralogy constraints in the presence of gene transfers. 2017. arXiv 1705.01240.

[16] M. Lafond and M. Hellmuth. Reconstruction of time-consistent species trees. Alg. Mol. Biol., $15: 16,2020$.

[17] N. Nøjgaard, M. Geiß, D. Merkle, P. Stadler, N. Wieseke, and M. Hellmuth. Time-consistent reconciliation maps and forbidden time travel. Alg. Mol. Biol., 13:2, 2018.

[18] D. Schaller, M. Hellmuth, and P. F. Stadler. A linear-time algorithm for the common refinement of rooted phylogenetic trees on a common leaf set. 2021. submitted; arXiv 2107.00072.

[19] C. Semple and M. Steel. Phylogenetics. Oxford University Press, Oxford UK, 2003.

[20] A. Tofigh, M. Hallett, and J. Lagergren. Simultaneous identification of duplications and lateral gene transfers. IEEE/ACM Trans. Comp. Biol. Bioinf., 8(2):517-535, 2011. 